\title{
Perfil epidemiológico, clínico y funcionalidad alcanzada de la población con lesión medular traumática asistida en el Servicio de Rehabilitación y Medicina Física en el Hospital Universitario
}

Clinical and epidemiological profile and functionality achieved in patients with traumatic spinal cord injury assisted at the Rehabilitation and Physical Medicine Service at the University Hospital Perfil epidemiológico, clínico e funcional alcançado da população com lesão medular traumática atendida no Serviço de Reabilitação e Medicina Física do Hospital Universitário

Dras. Marta Arriola ${ }^{1}$, Leticia López², Teresa Camarot³

\section{Resumen}

La lesión medular traumática puede afectar todas las funciones de la médula espinal determinando limitaciones de la actividad y restricciones en la participación. Conocer el perfil epidemiológico propio es imperativo para planificar la asistencia, los recursos y actuar en prevención. En la Cátedra de Rehabilitación hay una larga trayectoria de asistencia en equipo interdisciplinario a personas con lesión medular traumática; a partir de 1987 se documentaron datos que fueron analizados y presentados en 2004 en el Congreso Nacional de Neurología.

Objetivo: describir las características sociodemográficas, clínicas, funcionales y terapéuticas de nuestra población.

Método: se realizó un estudio longitudinal, retrospectivo, descriptivo y analítico de toda la población asistida durante el período 2004-2017. Resultados: $n=70,86 \%$ masculino, de $40 \pm 18$ años. Las causas fueron: precipitación $36,4 \%$, tránsito $31,8 \%$ y violencia 28,8\%; el nivel cervical $57 \%$, dorsal $40 \%$ y lumbar $3 \%$. La escala de la American Spinal Injury Association (ASIA) de inicio fue $36,2 \%$ A, 13,8\% B, 22,4\% C y 5,2\% D. ASIA final, 29,0\% A, 3,2\% B, 27,4\% C, 25,8\% D. El 45\% logró independencia en actividades básicas de la vida diaria, el $60 \%$ independencia en transferencias y el $45 \%$ en marcha.

Conclusiones: disminuyó el número de nuevos casos en los últimos cuatro años y cambió el perfil etiológico. Predominaron los hombres jóvenes, lesiones por precipitación, tránsito y violencia, las cuadriparesias incompletas, seguido de paraplejias completas e incompletas. Las complicaciones más frecuentes fueron nefrourológicas y escaras. De la funcionalidad alcanzada, la mayoría de los pacientes fueron dependientes en actividades de vida diaria, transfirieron en forma independiente y no lograron marcha.

\begin{tabular}{|c|c|c|c|}
\hline Palabras clave: & $\begin{array}{l}\text { Traumatismos de la médula espinal } \\
\text { Rehabilitación } \\
\text { Etiología } \\
\text { Epidemiología }\end{array}$ & Key words: & $\begin{array}{l}\text { Spinal cord injuries } \\
\text { Rehabilitation } \\
\text { Etiology } \\
\text { Epidemiology }\end{array}$ \\
\hline
\end{tabular}

1. Prof. Agregada, Cátedra de Rehabilitación y Medicina Física.

2. Prof. Adj. Cátedra de Rehabilitación y Medicina Física.

3. Prof. Cátedra de Rehabilitación y Medicina Física.

Departamento y Cátedra de Rehabilitación y Medicina Física, Facultad de Medicina, Universidad de la República, Hospital de Clínicas.

Correspondencia: Dra. Marta Arriola. Correo electrónico: martarriola7@gmail.com.

Los autores declaran no tener conflictos de intereses

Aprobado por el Comité de Ética de Investigación del Hospital de Clínicas.

Recibido: 11/9/2020

Aprobado: 13/1/2021

Attribution-NonCommercial 4.0 International (CC BY-NC 4.0) 


\section{Introducción e importancia del tema}

La lesión medular traumática (LMT) es un evento potencialmente catastrófico, pudiendo afectar todas las funciones de la médula espinal: motora, sensitiva y autonómica, determinando limitaciones de la actividad y restricciones en la participación. Impacta en la familia y en la sociedad con altos requerimientos asistenciales en agudo y a largo plazo (recursos humanos especializados, tiempos prolongados de tratamiento, etc.) y elevados costos directos e indirectos. Afecta fundamentalmente a personas en edad activa, aunque se asiste a un aumento de la edad de ocurrencia, vinculado al envejecimiento poblacional y a la etiología aumentando la frecuencia por caídas y precipitaciones $^{(1)}$. La incidencia anual mundial es variable, desde 49,1 por millón de habitantes en Nueva Zelanda a 10,0 por millón en Fiji, 8,0 por millón en España, y en Brasil varía entre 16 y 26 por millón ${ }^{(2)}$. La prevalencia es mayor en Estados Unidos (906 por millón) y menor en Francia (250 por millón) ${ }^{(3)}$. En Uruguay no contamos con datos epidemiológicos nacionales.

El abordaje por un equipo especializado mejora la calidad de la atención, disminuyendo la morbimortalidad (manejo adecuado y prevención de complicaciones) a corto y largo plazo ${ }^{(4)}$.

El departamento y Cátedra de Rehabilitación y Medicina Física (CRMF) está inserto en el Hospital Universitario, único en el país, siendo éste predominantemente de agudos, de alta complejidad, de referencia nacional y de rehabilitación ${ }^{(5)}$. Es un servicio polivalente donde se atienden pacientes con patologías viscerales, osteotendinomusculares y neurológicas pasibles de atención en rehabilitación, en diferentes etapas evolutivas; ya sea internados o ambulatorios, derivados desde la internación, o de otros centros asistenciales de nivel primario, secundario o terciario. Se realiza la capacitación de recursos humanos especializados, específicamente médicos residentes y posgrados en rehabilitación y medicina física; a nivel de grado realizan una materia optativa estudiantes de la Carrera de Medicina y de la Escuela Universitaria de Tecnología Médica, de disciplinas afines como terapia ocupacional, fisioterapia, neurofisiología y fonoaudiología. En la CRMF hay una larga trayectoria de asistencia en equipo interdisciplinario de rehabilitación a personas con LMT. A partir de 1987 se documentan datos que fueron analizados y presentados en 2004 en el Congreso Nacional de Neurología ${ }^{(6)}$.

En Estados Unidos, el National Spinal Cord Injury Statistical Center (NSCISC) publica anualmente datos nacionales demográficos y clínicos de las personas con $\mathrm{LMT}^{(7)}$. En nuestro medio las publicaciones encontradas se refieren a LM de diversas causas o en el contexto de ingresos por patología traumática en general ${ }^{(8)}$.
Conocer el perfil epidemiológico propio es un imperativo para planificar la asistencia, los recursos destinados y para identificar las causas que permitan actuar en prevención, dado que hasta la actualidad no existe "cura" luego del neuroagravio. Por otra parte, permite actuar en prevención secundaria y terciaria al identificar cuáles son las complicaciones más frecuentes en nuestra población.

Es objetivo principal de este estudio fue conocer las características sociodemográficas, clínicas y la funcionalidad de la población de lesionados medulares traumáticos asistida por el equipo de rehabilitación de la CRMF.

\section{Material y método}

Se realizó un estudio longitudinal, retrospectivo, descriptivo y analítico de toda la población asistida en el Hospital de Clínicas durante el período 2004-2017. Los datos se obtuvieron de las historias clínicas realizadas por el equipo de neurorrehabilitación. Los pacientes fueron captados en distintas etapas evolutivas de su enfermedad y provenientes de diferentes centros asistenciales del país.

Las variables utilizadas fueron las demográficas, año de lesión, etiología, nivel, severidad, asociación lesional, complicaciones médicas, tratamiento quirúrgico y funcionalidad alcanzada.

Para definir nivel y severidad de la lesión neurológica se utilizó la escala de la American Spinal Injury Association (ASIA), versión 2002; permite clasificar las lesiones de origen traumático en forma estandarizada y univer$\mathrm{sal}^{(9)}$, teniendo además valor pronóstico de recuperación funcional ${ }^{(10)}$. Las lesiones que fueron catalogadas como incompletas, pero no clasificadas según ASIA, se denominaron incompletas no especificadas (NE).

La funcionalidad se dividió en actividades de la vida diaria elementales (AVDE) y movilidad. Las AVDE evaluadas fueron alimentación, higiene y vestimenta. Se calificaron como independiente o dependiente. De la movilidad se evaluaron las transferencias y marcha. Las transferencias se calificaron como dependiente o independiente y la marcha como lograda o no.

Se realizó un análisis descriptivo, las variables cualitativas se describieron a través de sus frecuencias absolutas y relativas y las variables cuantitativas a través de media y desvío. Se compararon los datos obtenidos con el estudio previo del servicio y con el NSCISC.

El estudio fue aprobado por el Comité de Ética del Hospital Universitario.

\section{Resultados}

Se asistieron 70 pacientes, 63 nuevos casos y 7 con lesiones anteriores al 2004 que concurrieron a seguimien- 


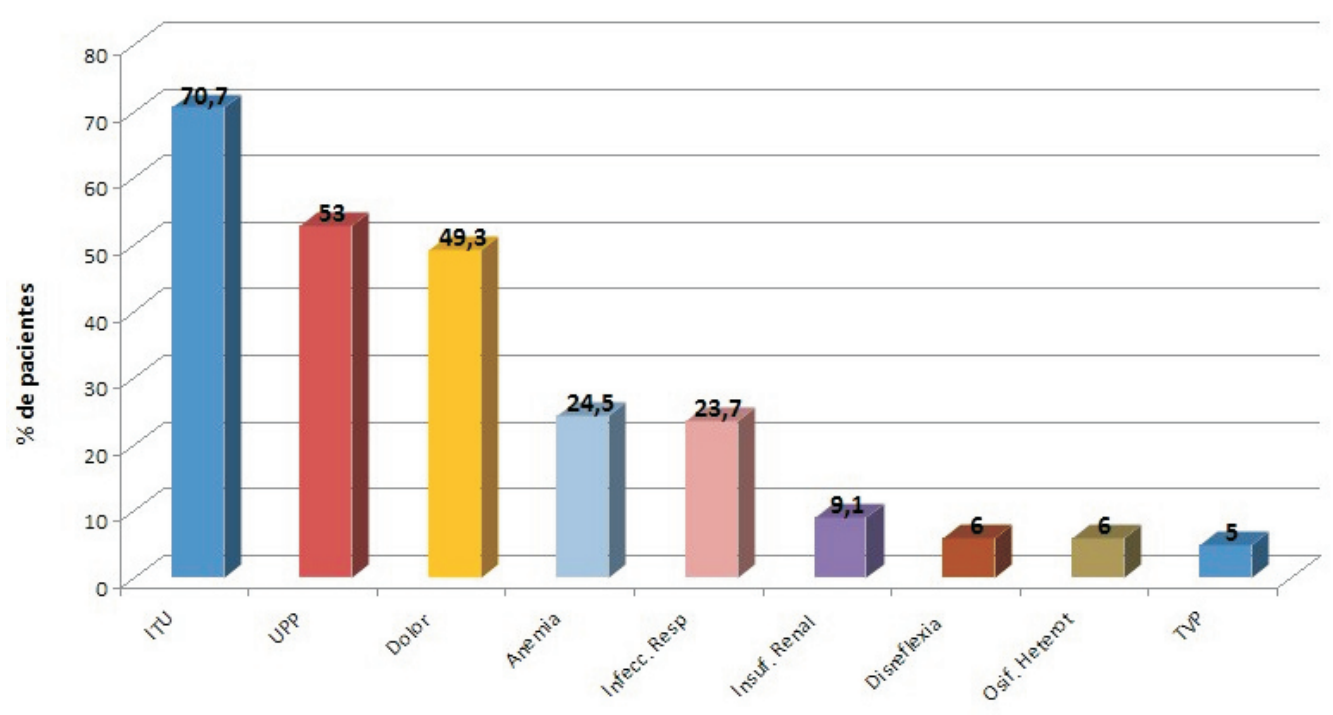

Figura 1. Complicaciones en pacientes con LMT, Hospital de Clínicas, 2004-2017.

to. Las características sociodemográficas y clínicas, así como la severidad lesional catalogada a través de ASIA de inicio y final, se representan en la tabla 1. Las complicaciones presentadas en el transcurso del proceso de atención se distribuyeron según lo muestra la figura 1. Con respecto a la vejiga neurógena y al vaciamiento vesical, la forma de drenaje más frecuente fue la micción espontánea $(47,6 \%)$, seguida del auto o hetero cateterismo intermitente limpio $(30,2 \%)$ y de la SV a permanencia $(22,2 \%)$. La funcionalidad en AVDE se registró solo en aquellos sujetos en los que se pudo obtener todos los datos requeridos ( $83 \%$ de nuestra población, $n=58)$. Respecto a las AVD elementales: $76 \%(n=44)$ logró independencia en alimentación, $53 \%(\mathrm{n}=34)$ en higiene básica, $66 \%(\mathrm{n}=38)$ en vestido tren superior y $45 \%$ $(n=26)$ en vestido tren inferior. En el último contacto con el equipo de rehabilitación, $45 \%$ de los sujetos eran independientes en todas las variables consideradas como AVD elementales, mientras que 55\% era dependiente en al menos una de las variables consideradas.

Respecto a la movilidad, $60 \%$ logró transferir en forma independiente y $45 \%$ logró la marcha.

\section{Discusión y análisis}

Nuevos casos anuales: el promedio de nuevos casos al año fue de 4,5; inferior al promedio de 5,8 obtenido en el trabajo realizado por Borelli y colaboradores en 1987-2003 en nuestro servicio. Nuestros datos muestran un descenso progresivo en el número de casos, pasando de 28 pacientes en los primeros cuatro años (2004 a 2007) a 10 en los últimos cuatro años (2014-2017); lo que corresponde de 7 a 2,5 nuevos casos/año (figura 2).
Pensamos que este cambio pueda deberse a que los pacientes estén siendo derivados a otros centros, ya que durante los primeros cuatro años que se analizan el único equipo de rehabilitación interdisciplinario en el país para pacientes complejos (entre ellos LMT) funcionaba en el Hospital de Clínicas. Es a partir de 2007 y 2010 que comienzan su funcionamiento y a recibir pacientes con LMT dos centros de rehabilitación: en Montevideo la "Casa de Gardel" y en Maldonado el "Centro Rehabilitación Física Maldonado-Cerema", respectivamente.

Edad: en comparación al estudio anterior en el mismo servicio, en que la edad promedio fue 29,5 años, actualmente es de 39,8 años, más cercana a la edad promedio de países desarrollados. El grupo etario entre 14 y 30 años fue el de mayor frecuencia, al igual que lo reportado internacionalmente, pero a diferencia de éstos, no se constata una distribución bimodal de la edad, estando ausente el otro pico en los $\geq 65$ años $^{(11)}$. El número de casos disminuyó con la edad.

Sexo: si bien predominó en el sexo masculino, como en todas las series internacionales, el ratio $\mathrm{H} / \mathrm{M}$ es de 6 , mayor que el obtenido en la revisión anterior en nuestro servicio $(4,8)$ y mayor que el reportado por NSCISC (año 2017) de 3,5, siendo el ratio en población general de ambos países muy semejante $(0,94 / 1$ en Uruguay y 0,97/1 en Estados Unidos).

Procedencia geográfica: aproximadamente la mitad de los pacientes proviene de Montevideo y la otra mitad del interior del país, tal como se distribuye la población de Uruguay. No se observaron diferencias con respecto al estudio previo. 


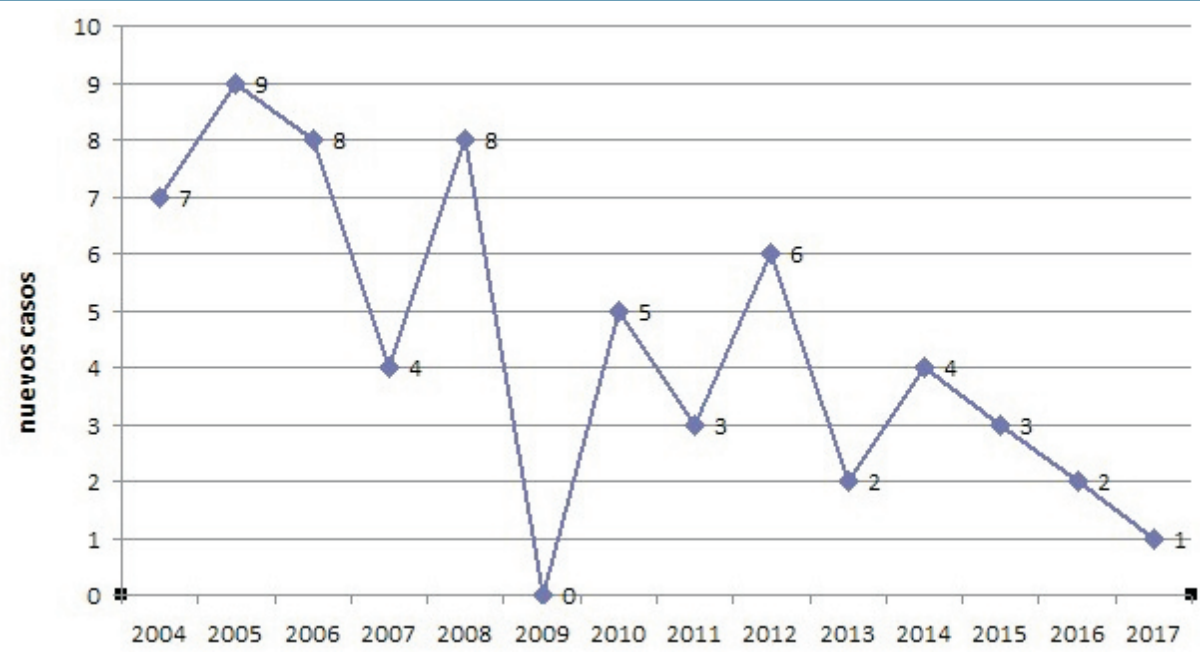

Figura 2. Nuevos casos por año. HC, 2004-2017.

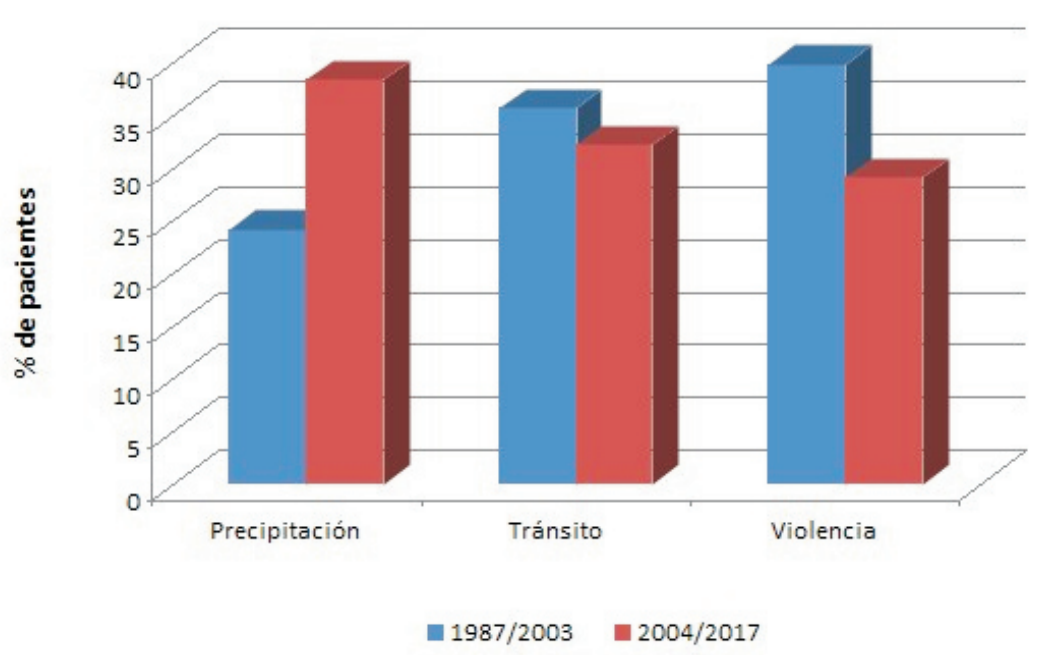

Figura 3. Distribución por etiologia de la lesión medular traumática. Comparativa 1987/2003 y 2004/2017

Etiología: la precipitación fue la causa más frecuente, seguida por tránsito y violencia; en el estudio previo la causa más frecuente fue violencia, seguida de tránsito y precipitación, aumentaron así las lesiones por precipitación y disminuyeron por tránsito y violencia. En la mayoría de las series de países desarrollados, incluido Estados Unidos, predominan las lesiones por tránsito y precipitación (figura 3).

Asociación de etiología con edad: en los menores de 30 años $(\mathrm{n}=27)$ predominó la violencia $(52 \%)$, seguido de tránsito (26\%) y precipitación (15\%); en los mayores de 31 años $(\mathrm{n}=43)$ predominó precipitación (46,5\%), seguido de tránsito (32,5\%) y violencia (12\%). Esta distribución concuerda con datos internacionales. En los menores de 30 años predomina tránsito o violencia según el grado de desarrollo país, así como en la población adulta predomina la precipitación.

Asociación de etiología con sexo: en el hombre predominó la precipitación, seguido de tránsito y violencia. En tanto que en la mujer fue tránsito, violencia y precipitación.

No se encontró asociación entre etiología y severidad, ni etiología y nivel.

En cuanto al nivel lesional predominaron las lesiones cervicales, seguidas de las dorsales (tabla 1), así como lo muestran los datos internacionales ${ }^{(12)}$. Llama la atención en nuestro estudio el alto índice de cervicales altos (occipito atloidea hasta C4) (40\% del total de las lesiones cervicales).

Asociación entre nivel y completitividad: en las lesiones cervicales predominaron las lesiones incomple- 
Tabla 1. Caracteristicas sociodemográficas y clinicas de la población asistida con LMT, HC, 2004-2017

\begin{tabular}{|c|c|c|}
\hline \multirow[t]{3}{*}{ Edad } & Media & 39,8 años \\
\hline & Desvío & 17,7 años \\
\hline & Mediana & 35 años \\
\hline \multirow[t]{2}{*}{ Sexo } & Masculino & $86 \%(n=60)$ \\
\hline & Femenino & $14 \%(n=10)$ \\
\hline \multirow[t]{2}{*}{ Procedencia } & Interior & $55 \%(n=38)$ \\
\hline & Capital & $45 \%(n=31)$ \\
\hline \multirow[t]{5}{*}{ Etiología } & Precipitación & $36,4 \%(n=24)$ \\
\hline & Tránsito & $31,8 \%(n=21)$ \\
\hline & Violencia & $28,8 \%(n=19)$ \\
\hline & Zambullida & $1,5 \%(n=1)$ \\
\hline & Otros & $1,5 \%(n=1)$ \\
\hline \multirow[t]{3}{*}{ Nivel } & Cervicales & $57 \%(n=40)$ \\
\hline & Dorsales & $40 \%(n=28)$ \\
\hline & Lumbares & $3 \%(n=2)$ \\
\hline \multirow[t]{5}{*}{ Lesiones asociadas } & TEC & $31 \%(n=22)$ \\
\hline & Tórax & $17 \%(n=12)$ \\
\hline & Abdomen & $8,6 \%(n=6)$ \\
\hline & Miembros & $4,3 \%(n=3)$ \\
\hline & Plexo & $7,1 \%(n=5)$ \\
\hline Lesión raquídea & $69 \%(n=45)$ & \\
\hline Cirugía & $41 \%(n=27)$ & \\
\hline \multirow[t]{5}{*}{ ASIA inicio } & A & $36,2 \%(n=21)$ \\
\hline & B & $13,8 \%(n=8)$ \\
\hline & C & $22,4 \%(n=13)$ \\
\hline & D & $5,2 \%(n=3)$ \\
\hline & Incompleta NE & $22,4 \%(n=13)$ \\
\hline \multirow[t]{5}{*}{ ASIA final } & A & $29,0 \%(n=18)$ \\
\hline & B & $3,2 \%(n=2)$ \\
\hline & C & $27,4 \%(n=17)$ \\
\hline & D & $25,8 \%(n=16)$ \\
\hline & Incompleta NE & $14,5 \%(n=9)$ \\
\hline
\end{tabular}

tas y en las dorsales las completas, al igual que en los reportes internacionales, lo que se explica por la neuroanatomía del canal raquimedular (figura 4).

\section{Evolución según ASIA}

Para realizar el análisis solo se incluyeron los pacientes catalogados por ASIA al inicio y final. La valoración del ASIA fue realizada en distintos momentos evolutivos y por evaluadores diferentes. El 85,7\% de los sujetos ASIA A se mantuvieron como A, de modo que 14,3\% mejoraron su clasificación de competitividad, menor a reportes internacionales. El 4,8\% de los ASIA A pasaron a B, también menor a lo reportado ${ }^{(13,14)}$. El $87,5 \%$ de los ASIA B mejoraron, pasando en su mayoría a ASIA C. Los ASIA B se distribuyeron por igual en cervicales y torácicos, siendo además un $n$ muy bajo como para poder analizar este dato. No tenemos suficiente $n$ en esta población.

El 50\% de los ASIA C mejoraron a D (tabla 2). En la población estudiada confirmamos que la severidad inicial fue un predictor primario de recuperación neurológica.

Evolución del ASIA según el nivel lesional. Observamos que en los cervicales, $71,4 \%$ de los ASIA A se mantuvo como $\mathrm{A}$, ninguno pasó a $\mathrm{B}$ y $14,3 \%$ pasó a $\mathrm{C}$ y D. La menor conversión de los ASIA A en incompletos, en comparación a lo reportado internacionalmente, podría vincularse a errores en el examen, donde quizás algunos de los catalogados inicialmente como completos eran realmente $\mathrm{B}$, lo cual explicaría también el alto porcentaje de los que pasaron a C y D. Por otro lado, pensamos que el bajo porcentaje de descompresión y fijación quirúrgica inicial podría en parte explicar esta distribución.

En el caso de los torácicos, $92,9 \%$ de los ASIA A se mantuvo igual; 7,1\% pasó a $\mathrm{B}$ y ninguno pasó a $\mathrm{C}$ o $\mathrm{D}$. La menor conversión de completos en incompletos que lo reportado internacionalmente podría vincularse también a errores en la clasificación por ASIA y al tratamiento quirúrgico conservador ${ }^{(15)}$.

No se observó asociación entre etiología y recuperación por ASIA.

\section{Lesión vertebral}

El $68,75 \%$ de los pacientes presentó lesión raquídea frente al 80\% referido en NSCISC. Encontramos 31\% de SCIWORA ${ }^{(16)}$, mayor a lo reportado internacionalmente de $9 \%$ a $14 \%$ y en la NSCISC, creemos que esto es en parte explicable porque en esa muestra predominan los accidentes de tránsito.

Tratamiento quirúrgico: fueron intervenidos $41 \%$ vs $79,3 \%$ en NSCISC, que operaron el $99,1 \%$ de los pacientes con lesiones vertebrales, mientras que en nuestra 


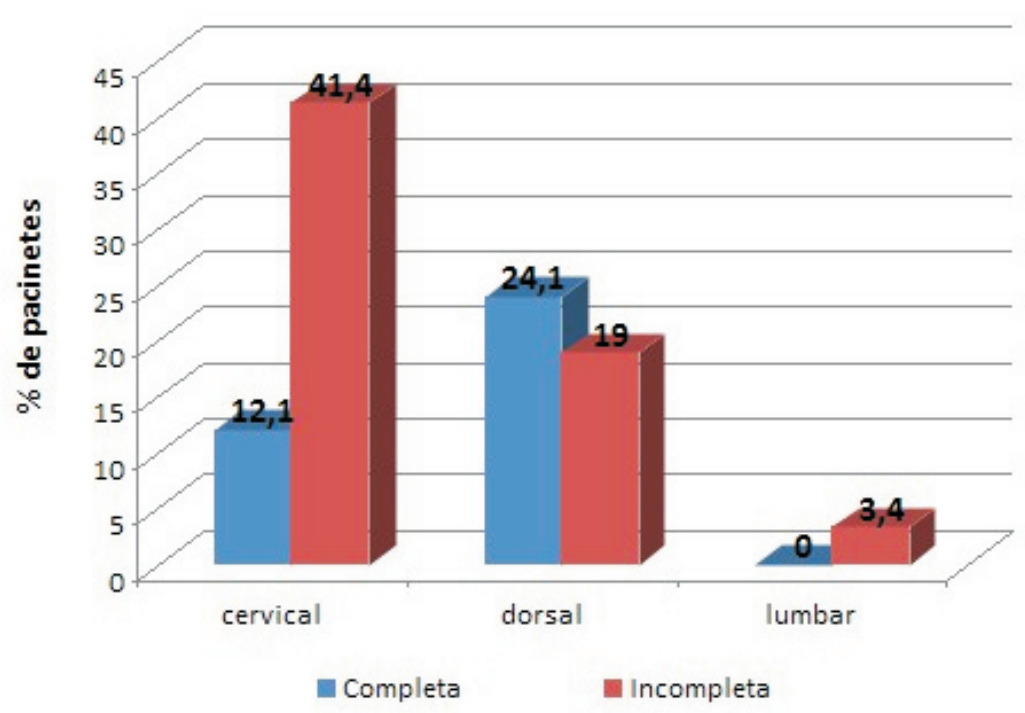

Figura 4. Distribución según nivel y completitividad, LMT, HC, 2004-2017

Tabla 2. Evolución según ASIA de los LMT, HC, 2004-2017

\begin{tabular}{lcc}
\hline & ASIA inicio & ASIA final \\
& $\%$ ptes \\
\hline A & $85,7 \%$ A \\
& $4,8 \%$ B \\
& $4,8 \%$ C \\
& $4,8 \%$ D \\
\hline B & $12,5 \%$ B \\
& $50 \%$ C \\
& $37,5 \%$ D \\
\hline C & $50 \%$ C \\
& $50 \%$ D \\
\hline
\end{tabular}

población recibieron cirugía el $72 \%$ de ellos. De los pacientes con lesión medular sin lesión raquídea, se operó el $5 \%$.

Lesiones asociadas: la frecuencia de lesiones asociadas fue de $44 \%$, igual al trabajo anterior del servicio $(43,9 \%)$ y mayor al encontrado en NSCISC 2017 de $38,4 \%$.

Complicaciones: las más frecuentes fueron las infecciones urinarias y las lesiones de piel, similar a la serie anterior. La baja de frecuencia de complicaciones respiratorias podría estar vinculada a que no todos los pacientes se atendieron en etapa aguda en nuestro centro asistencial y al abordaje precoz para reeducación respiratoria e higiene canalicular durante el proceso de atención. La baja ocurrencia de disreflexia autonómica (6\%) está seguramente vinculada al subregistro. El 4,8\% presentó TVP, sensiblemente menor a la del trabajo anterior del servicio $(9,1 \%)$, posiblemente en relación con el estricto protocolo de tromboprofilaxis puesto en marcha en nuestro hospital; sin embargo, sigue siendo mayor a lo reportado internacionalmente.

Vejiga neurógena y vaciamiento vesical: en 12,1\% de los pacientes su vaciamiento fue por sonda permanente, lo que podría estar vinculado al insuficiente apoyo de cuidadores, bajo nivel socioeconómico de la población que se atiende en nuestro hospital y pobre accesibilidad a las sondas de drenaje para cateterismo intermitente, entre otros factores.

Con respecto al porcentaje de pacientes con micción espontánea, pensamos que no solo está vinculado a la reeducación vesical, sino también a pacientes que teniendo indicación de CIL, optan por no hacerlo aun en detrimento de su aparato urinario alto.

\section{De la funcionalidad}

AVDE según nivel: lograron independencia en alimentación todos los dorsolumbares y $53 \%$ de los cervicales. De los cervicales que no lograron independencia en alimentación, 50\% fueron altos. Lograron independencia en higiene básica el $37,5 \%$ de los cervicales y $71 \%$ de los dorsales.

Transferencias: observamos que la independencia en transferencias es mayor en los más jóvenes (figura 5). Cuanto más alto el nivel, menor capacidad de transferir 


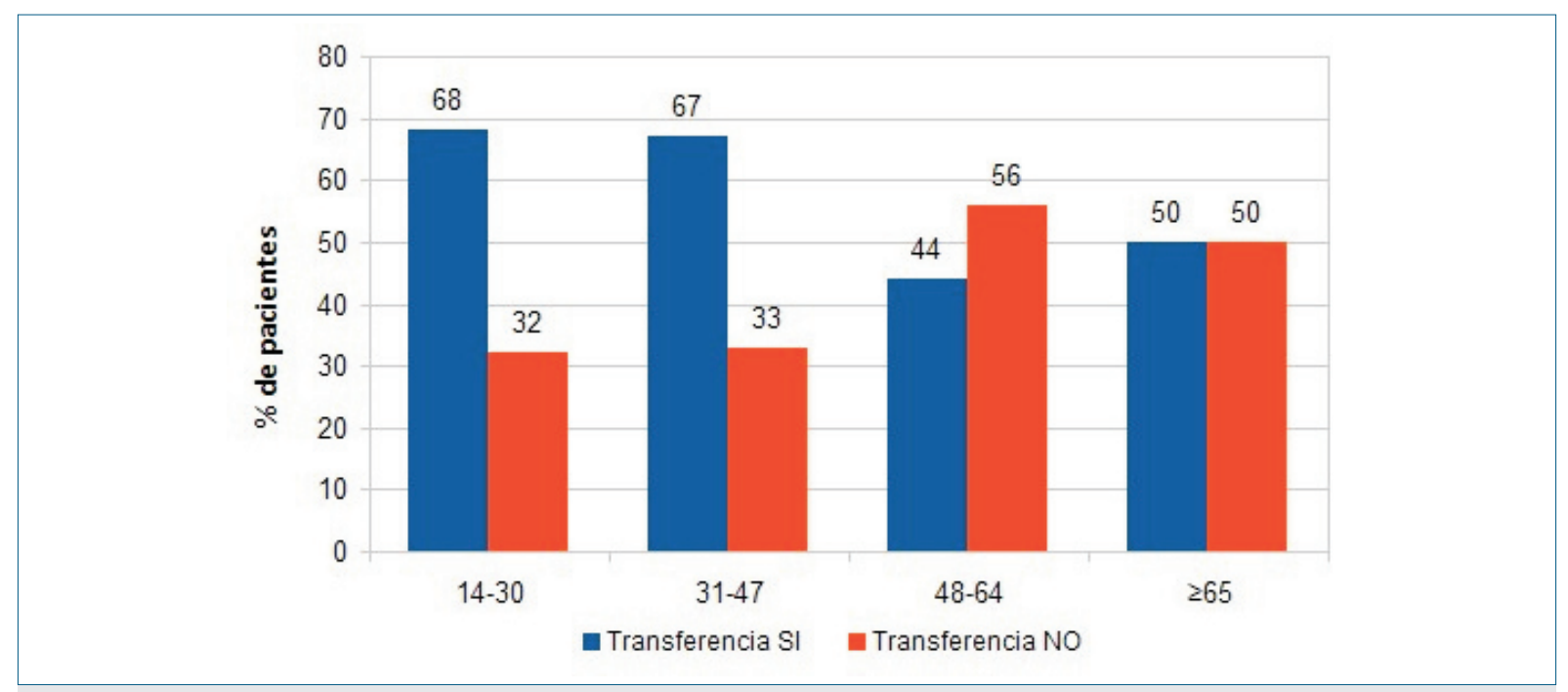

Figura 5. independencia en transferencias según edad, LMT, HC,2004-2017.

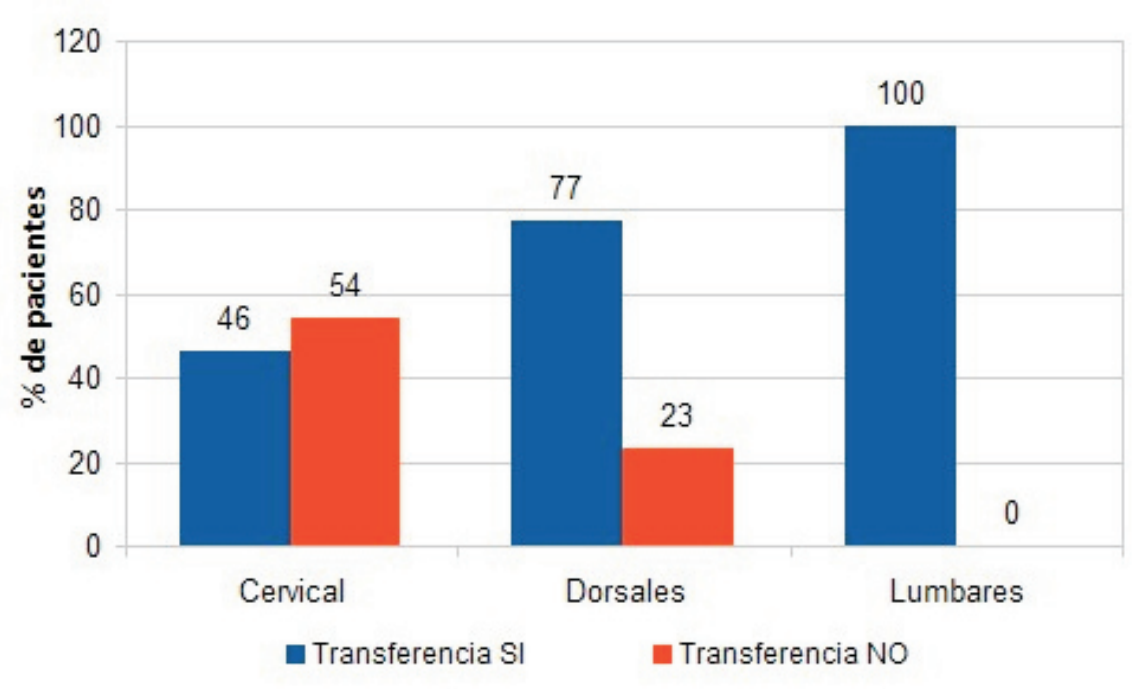

Figura 6. Transferencias según nivel. LMT, HC, 2004-2017.

(figura 6). Marcha según edad: en los $<65$ años no se evidencia relación entre la edad y la capacidad de deambular. En los $\geq 65$ años predominan los no deambuladores.

Marcha según nivel: de los sujetos que lograron la marcha, $72 \%$ fueron cervicales, $21 \%$ torácicos y $7 \%$ lumbares. Todos fueron incompletos. El 100\% de los lumbares lograron la marcha y $58 \%$ de los cervicales, mientras que solo $22 \%$ de los dorsales lo alcanzaron. Marcha según ASIA final: como es esperable, el 100\% de A y B no lograron marcha. De los ASIA C, $75 \%$ no logró marcha, siendo $67 \%$ de ellos cervicales o torácicos altos $(\leq \mathrm{T} 6)$.

\section{Conclusiones}

Se confirma la percepción clínica de una tendencia a la baja en las consultas por LMT en el Hospital Universitario en los últimos años, más marcada en los últimos cuatro años. La edad promedio se asemejó a lo observado en los países desarrollados. Se observó un descenso de la incidencia de las LMT con la edad. De la etiología, el orden de frecuencia fue precipitación, tránsito y violencia, cambiando el perfil con respecto al estudio previo de nuestro servicio. Observamos una incidencia mayor de SCIWORA que en los reportes internacionales. Las complicaciones médicas más frecuentes fueron nefrourológicas, escaras y dolor. Destacamos la baja inci- 
dencia de TVP. Lograron la independencia en AVDE el $44 \%$ de los pacientes. Todos los que lograron marcha fueron incompletos.

\section{Consideraciones finales}

En cuanto a las limitaciones de nuestro estudio, destacamos que las evaluaciones mediante escalas fueron tomadas en momentos diferentes, hubo baja adherencia con el consiguiente pobre seguimiento; derivaciones en etapa evolucionada y de hospitales generales.

Comprobamos que los pacientes desarrollan más complicaciones, no siempre alcanzan los objetivos potenciales acordes a la persona y a la lesión, y el abordaje quirúrgico es menor al de reportes internacionales.

La lesión medular implica afectación multisistémica que requiere de un abordaje interdisciplinario, por parte de recursos humanos con experticia, que permitan llevar adelante un programa de rehabilitación adecuado y oportuno, desarrollando al máximo las potenciales capacidades.

Creemos de fundamental importancia que existan centros de rehabilitación de referencia regional o nacional de atención de estos pacientes desde la etapa aguda y seguimiento a largo plazo, así como una base de datos nacional.

\section{Summary}

Traumatic spinal cord injury may affect all functionalities of the spinal cord, what determines limitation in activity and restrictions in participation. Learning about its epidemiological profile is of the essence to plan healthcare, resources and preventive actions. The Rehabilitation Chair has a long history in providing healthcare to people with traumatic spinal cord injury in multidisciplinary teams. Data has been recorded since 1987, which information was analysed and presented at the National Neurology Conference in 2004.

Objective: to describe the socio-demographic, clinical, functional and therapeutic characteristics of our population.

Method: we conducted a longitudinal, retrospective, descriptive and analytical study of the entire population assisted between 2004 and 2017.

Results: $\mathrm{n}=70,86 \%$ male, $40 \pm 18$ years old. The following causes were found: falls $36.4 \%$, road accidents $31.8 \%$ and violence $28.8 \%$; cervical level $57 \%$, dorsal spine $40 \%$ and lumbar spine $3 \%$. Initial ASIA impairment scale was $36.2 \% \mathrm{~A}, 13.8 \% \mathrm{~B}, 22.4 \% \mathrm{C}$ and $5.2 \%$ D. Final ASIA impairment scale was $29.0 \%$ A, $3.2 \%$ B, $27.4 \%$ C, $25.8 \%$ D. $45 \%$ achieved independence in everyday basic activities, $60 \%$ transfer independence and $45 \%$ independent gait.
Conclusions: the number of new cases decreased in the last 4 years and the etiological profile changed. Young men prevailed, lesions caused by falls, road accidents and violence were the most frequent, incomplete quadriparesis, followed by complete and incomplete paraplegia. The most frequent complications were nephro-urological and pressure sores. As to the functionality achieved, most patients were dependent in every day life activities, they could transfer independently and could not achieve independent gait.

\section{Resumo}

A lesão medular traumática pode afetar todas as funções da medula espinhal, determinando limitações de atividade e restrições de participação. Conhecer o perfil epidemiológico da população atendida é imprescindível para planejar cuidados, recursos e atuar na prevenção. A Cátedra de Reabilitação (CRMF) tem uma longa trajetória de assistência com equipe interdisciplinar às pessoas com Lesão Medular Traumática; a partir de 1987, foram registrados dados que foram analisados e apresentados em 2004 no Congresso Nacional de Neurologia.

Objetivo: descrever as características sociodemográficas, clínicas, funcionais e terapêuticas da população atendida pela equipe de reabilitação da CRMF no período 2004-2007.

Métodos: foi realizado um estudo longitudinal, retrospectivo, descritivo e analítico de toda a população assistida no período de 2004-2017.

Resultados: 70 pacientes foram atendidos sendo $86 \%$ de sexo masculino, com idade $40 \pm 18$ anos. As causas foram: precipitação $36,4 \%$, trânsito $31,8 \%$ e violência $28,8 \%$; o nível cervical $57 \%$, dorsal $40 \%$ e lombar 3\%. A Escala da American Spinal Injury Association (ASIA) inicial era $36,2 \% \mathrm{~A}, 13,8 \% \mathrm{~B}$, $22,4 \%$ C e $5,2 \%$ D. ASIA final, $29,0 \%$ A, $3,2 \%$ B, $27,4 \%$ C, $25,8 \%$ D. $45 \%$ alcançaram independência nas atividades básicas de vida diárias, $60 \%$ independência nas transferências e $45 \%$ na marcha.

Conclusões: o número de casos novos diminuiu nos últimos 4 anos e o perfil etiológico mudou. Predominou o sexo masculino, as lesões por precipitação, trânsito e violência, as quadriparesias incompletas, seguidas de paraplegias completas e incompletas. As complicações mais frequentes foram nefrourológicas e escaras. Em relação à funcionalidade alcançada, a maioria dos pacientes era dependente nas atividades da vida diária, movia-se de forma independente e não conseguiram autonomia na marcha. 


\section{Bibliografía}

1. Kang Y, Ding H, Zhou HX, Wei ZJ, Liu L, Pan DY, et al. Epidemiology of worldwide spinal cord injury: a literature review. J Neurorestoratol 2018; 6:1-9.

2. Vieira R, Gianini L, Bastianello R, Arantes A. Epidemiology of traumatic spinal injuries in Brazil: systematic review. Arq Bras Neurocir 2014; 33(2):100-6.

3. Singh A, Lindsay T, Suhkvinder KR, Nouri A, Fehlings MG. Global prevalence and incidence of traumatic spinal cord injury. Clin Epidemiol 2014; 3:309-31.

4. Cardenas DD, Curt A. Rehabilitation in spinal cord injury. En: Selzer M, Clarke S, Cohen L, Duncan P, Gage F, eds. Textbook of neural repair and rehabilitation. New York: Cambridge University Press, 2006:615-36.

5. Uruguay. Universidad de la República. Rectoría. Ordenanza del Hospital de Clínicas "Dr. Manuel Quintela". Exp. N. ${ }^{\circ}$ 151100-0009600. Resol. n. ${ }^{\circ}$ 05-CDC-13/10/2015.

6. Borelli G, Arriola M, Bachetta L, Aguirrezabal X. El lesionado raquimedular traumático. Características epidemiológicas de la población asistida por el equipo de rehabilitación del Hospital de Clínicas. En: Sociedad Uruguaya de Neurología. II Congreso Nacional de Neurología. 8-11 de setiembre 2004. Colonia, Uruguay.

7. National Spinal Cord Injury Statistical Center. 2017 Annual statistical report for the spinal cord injury model systems public version. Birmingham, AL: University of Alabama at Birmingham, 2017.

8. Martínez F, Algorta M. Lesiones medulares por arma blanca. Presentación de casos clínicos y propuesta de manejo. Rev Urug Med Interna 2017; (1):39-48.
9. National Spinal Cord Injury Statistical Center. 2017 Annual statistical report for the spinal cord injury model systems public version. Birmingham, AL: University of Alabama, 2017.

10. van Middendorp JJ, Goss B, Urquhart S, Atresh S, Williams RP, Schuetz M. Diagnosis and prognosis of traumatic spinal cord injury. Global Spine J 2011; 1(1):1 ] 8 . doi:10.1055/s-0031-1296049.

11. van den Berg ME, Castellote JM, Mahillo-Fernandez I, de Pedro-Cuesta J. Incidence of spinal cord injury. Worldwide: a systematic review. Neuroepidemiology 2010; 34:184-92.

12. Kang Y, Ding H, Zhou H, Wei Z, Liu L, Pan D, et al. Epidemiology of worldwide spinal cord injury: a literature review. J Neurorestoratol 2017; 6:1-9. doi:10.2147/ JN.s143236.

13. Spiess MR, Müller RM, Rupp R, Schuld C, van Hedel HJ. Conversion in ASIA impairment scale during the first year after traumatic spinal cord injury. J Neurotrauma 2009; 26(11):2027-36.

14. Kirshblum SC, Botticello AL, Dyson-Hudson TA, Byrne R, Marino RJ, Lammertse DP. Patterns of sacral sparing components on neurologic recovery in newly injured persons with traumatic spinal cord injury. Arch Phys Med Rehabil 2016; 97(10):1647-55.

15. Lee BA, Leiby BE, Marino RJ. Neurological and functional recovery after thoracic spinal cord injury. J Spinal Cord Med 2016; 39(1):67-76.

16. Bazán PL. Significance of SCIWORA in adults. Coluna/Columna 2015; 14(2):134-7.

\section{Contribución de autoras}

Marta Arriola, Leticia López participaron en todas las etapas de elaboración del artículo.

Teresa Camarot participó del análisis, revisión crítica y redacción.

Leticia López, https://orcid.org/0000-0002-1762-2602

Marta Arriola, https://orcid.org/0000-0001-7948-4228

Teresa Camarot, https://orcid.org/0000-0001-5446-3383 